\title{
Patient specific modeling of the HPA axis related to clinical diagnosis of depression
}

Bangsgaard, Elisabeth; Ottesen, Johnny T.

Published in:

Mathematical Biosciences

DOI:

10.1016/j.mbs.2016.10.007

Publication date:

2017

Document Version

Early version, also known as pre-print

Citation for published version (APA):

Bangsgaard, E., \& Ottesen, J. T. (2017). Patient specific modeling of the HPA axis related to clinical diagnosis of depression. Mathematical Biosciences, 287, 24-35. https://doi.org/10.1016/j.mbs.2016.10.007

\section{General rights}

Copyright and moral rights for the publications made accessible in the public portal are retained by the authors and/or other copyright owners and it is a condition of accessing publications that users recognise and abide by the legal requirements associated with these rights.

- Users may download and print one copy of any publication from the public portal for the purpose of private study or research.

- You may not further distribute the material or use it for any profit-making activity or commercial gain.

- You may freely distribute the URL identifying the publication in the public portal.

\section{Take down policy}

If you believe that this document breaches copyright please contact rucforsk@kb.dk providing details, and we will remove access to the work immediately and investigate your claim. 


\title{
Patient specific modeling of the HPA axis related to clinical diagnosis of depression
}

\author{
Elisabeth O. Bangsgaard ${ }^{a}$ and Johnny T. Ottesen ${ }^{b *}$ \\ ${ }^{a}$ Department of Applied Mathematics and Computer Science, Technical University of Denmark, \\ 2800 Kgs. Lyngby, Denmark \\ ${ }^{b}$ Department of Science and Environment, Roskilde University, Building 27.1, 4000 Roskilde, \\ Denmark \\ *Corresponding author. E-mail address: johnny@ruc.dk
}

\begin{abstract}
A novel model of the hypothalamic-pituitary-adrenal axis is presented. The axis is an endocrine system responsible for coping with stress and it is likely to be involved in depression. The dynamics of the system is studied and existence, uniqueness and positivity of the solution and the existence of an attracting trapping region are proved. The model is calibrated and compared to data for healthy and depressed subjects. A sensitivity analysis resulting in a set of identifiable physiological parameters is provided. A subset is selected for parameter estimation and a reduced version of the model is stated and an approximated version is discussed. The model is physiologically based, thus parameters are representative for gland functions or elimination processes. Hence the model may be used for pointing out pathologies by parameter estimation and hypothesis testing whereby it may be used as an objective and refined method for diagnosing depression and suggesting individual treatment protocols. Finally, the method may inspire pharmaceutical companies to develop target specific psychopharmaca for more effective and individual treatment.
\end{abstract}

Keywords: Depression, HPA axis, Patient Specific, Mathematical Modeling, Non-Linear ODE Model, Parameter Estimation, Clinical Impact, Bio-marker.

\section{Introduction}

Depression is a mental disease diagnosed by psychiatrists. Such diagnoses are based on patient interviews and symptoms with uncertainties as high as $30 \%$ as a consequence [7]. However, it is commonly believed that depression is caused by 


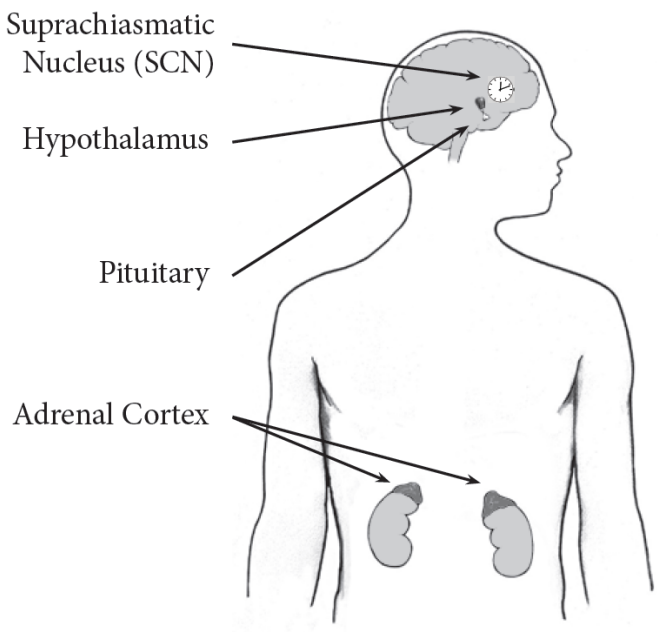

(a)

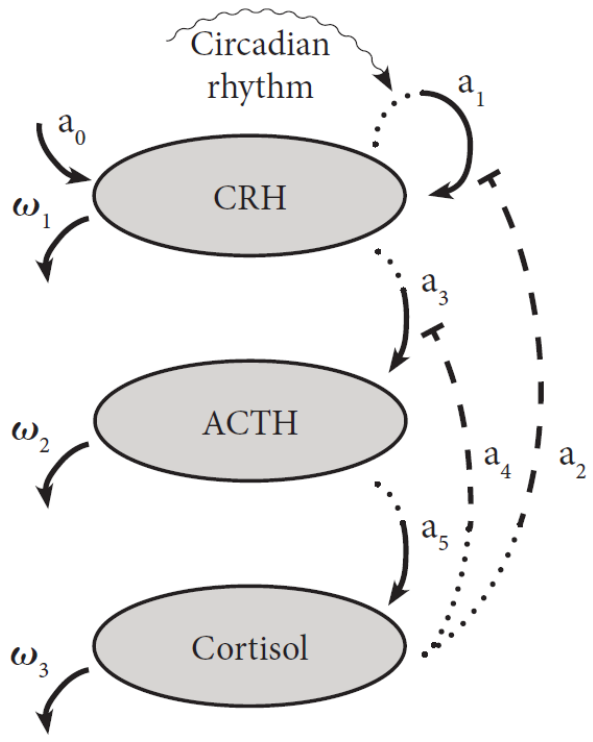

(b)

Figure 1: Illustration of the HPA axis. CRH released from hypothalamus stimulates the synthesis and release of ACTH in the pituitary. ACTH is transported to the adrenal cortex stimulating the synthesis and release of cortisol. Cortisol feeds back by inhibiting the synthesis of CRH and ACTH. The observed circadian rhythm in ACTH and cortisol is synchronized by the SCN. (a) Anatomy of the HPA axis. (b) Diagram of the dynamics of the model (1-4) in Section 3.2. The rates indicated at the figure agree with those in Equations (1-4) and are further discussed in Section 3.3.

malfunctions in the Hypothalamic-Pituitary-Adrenal (HPA) axis - a coupled endocrine system $[9,12,20,40]$. The HPA axis regulates the level of glucocorticoid hormones in the blood. The hormone cortisol is essential for maintenance of body homeostasis as a response to both mental and physical stress. It converts glycogen into glucose. Keeping the cortisol concentration within a certain range is important: A too high level of cortisol (hypercortisolism) can cause depression, diabetes, visceral obesity or osteoporosis if maintained over longer periods of time. A too low concentration (hypocortisolism) is not desirable either, since it can result in a disturbed memory formation or life-threatening adrenal crisis beyond depression. [11] Apart from this, the secretion and clearance of cortisol plays a role in the acute inflammatory response where it acts as an anti-inflammatory mediator in the system. [46, 48] The secretion of cortisol is regulated by a feedback system. Hippocampus stimulates hypothalamus to secrete corticotropin releasing 
hormone $(\mathrm{CRH})$ into the portal blood vessel of the hypophyseal stalk, where it is transported to the anterior pituitary stimulating the synthesis and release of adrenocorticotropic hormone (ACTH) into the systemic circulation. ACTH is transported by the blood circulation to all regions of the body especially to the adrenal cortex, where it stimulates the production and release of cortisol. Cortisol feeds back on the hypothalamus and inhibits synthesis of CRH and ACTH, leading to a negative feedback on cortisol. [18, 30, 41] The anatomy of the HPA axis is illustrated in Figure 1(a).

The secretion of cortisol has been studied in many cases revealing both circadian and ultradian oscillations in the concentration [8, 9, 10, 16, 41]. Also the release of ACTH follows similar patterns prior to that for cortisol by about 10 minutes. In humans, the circadian rhythm is observed as low concentrations of cortisol in the very early hours followed by an increase during the early morning hours. The cortisol level peaks around noon and returns toward the low level over the evening and early night. During most of the night cortisol fluctuates around the low level. The circadian rhythm is mainly synchronized by the circadian clock at the suprachiasmatic nucleus (SCN) located in the hypothalamus in the brain [1]. It is generally believed that the circadian pattern is modulated by exogenous factors such as daylight, temperature, psychological as well as physical stress, but that the ultradian pattern is caused by intrinsic dynamics of the HPA axis itself $[13,19,32,36,54]$. According to Chrousos [10], the frequency of the ultradian oscillations are rather insensitive to stress whereas the amplitude increases as a consequence of stress. A typical example of ACTH and cortisol data is shown in Figure 2 in Section 5.

The function of the HPA axis may be elegantly summarized by quoting Kurt Vonnegut Jr. whom in the novel Breakfast of Champions poetically wrote [51],

My mind sent a message to my hypothalamus, told it to release the hormone $C R F[=C R H]$ into the short vessels connecting my hypothalamus and my pituitary gland. The $C R F[=C R H]$ inspired my pituitary gland to dump the hormone ACTH into my blood stream. My pituitary had been making and storing ACTH for just such an occasion, and nearer and nearer the zeppelin came. And some of the $A C T H$ in my bloodstream reached the outer shell of my adrenal gland, which had been making and storing glucocorticoids for emergencies. My adrenal gland added the glucocorticoids to my bloodstream. They went all over my body, changing glycogen into glucose. Glucose was muscle food. It would help me fight like a wildcat or run like a deer. 
Understanding the interplay between the various elements of the HPA axis is interesting and important. Since several feedback mechanisms are working simultaneously in the HPA axis, cause and effect may be hard to distinguish. A mathematical model may help in understanding this and can be a useful tool for pointing out different ways in which malfunctioning may occur. More specifically, if we are able to estimate parameters based on a correct model of the HPA axis and individual data, parameters varying significantly between groups of depressed and normal subjects can possibly be identified. By measuring concentrations of the hormones ACTH and cortisol in blood plasma samples, such varying parameters characterize the state of the disease and at the same time pinpoint the mechanisms which are malfunctioning.

\section{Previous mathematical modeling of the HPA axis}

Early attempts of establishing objective markers for diagnosing depression based on hormone levels and patterns produced by the HPA axis have not been successful [9, 12, 43]. Among such attempts are ultradian oscillation pattern [43], mean concentration of cortisol and ACTH [9, 12, 43, 39] and approximated entropy [9]. Only in one recent case a statistically significance marker was found. In [39] a mixture density function and cluster analysis approach are showing that the outcome of three clusters turn out to be statistically related to non-depressed subjects, hypercortisolemic depressed subjects and hypocortisolemic depressed subjects. It is shown that healthy subjects may have an elevated (suppressed) level of cortisol or ACTH, however, the healthy system is able to deal with such elevated (suppressed) levels by compensating through suppressing (stimulating) the other component. Depressed subjects, however, are incapable of making such compensation.

It is commonly known that cortisol inhibits the secretion of CRH through glucocorticoid receptors (GR) situated in the hypothalamus [52]. In addition, cortisol also performs a negative feedback on the secretion of ACTH through GR situated in pituitary [47]. This description is called 'the minimal model' of the HPA axis and has been thoroughly investigated in [50]. In [6, 24, 27, 28, 31, 44, 45, 50] the splitting of the circadian and ultradian rhythms is assumed in such a way that the ultradian rhythm is considered an inherent behavior of the HPA axis whereas the circadian rhythm is thought of as an external input to the axis. All of these models consists of systems of coupled non-linear ordinary differential equations. Despite differences in their approach, the common aim was to examine the system for persistent oscillations. In [44, 45, 50] this was done by looking for a possible 
Hopf-bifurcation of stable fixed points guaranteeing oscillating solutions. However, as documented in [50] such 'minimal model' of the HPA axis is not capable of reproducing the characteristics seen in data using reasonable parameter values. This suggests that persistent ultradian oscillations arise from other mechanisms $[26,25,49]$. It has been suggested that the ultradian oscillations may arise from the introduction of a time delay $[4,50]$. However, it is mathematically easy to impose oscillations by introducing time-delays and investigations show that rather large time-delays are needed (i.e. at least 18 minutes) compared to well-known mechanisms [50]. Furthermore, it has been suggested that the ultradian oscillations are imposed from outside. A last possibility is that something is missing in the minimal description of the HPA axis. In the latter case, we have suggested the inclusion of mechanisms from hippocampus [2, 3].

In the work of Jelić et al. [24], it is assumed that changes in the dynamics of $\mathrm{CRH}$ are negligible and thus large time-delays produce ultradian rhythms. The circadian rhythm of cortisol is modeled as an external periodic function while the model is not calibrated to data and the concentration of $\mathrm{CRH}$ is considered to be without influence. Conrad et al. [11] lump CRH and ACTH into one variable. While the CRH-ACTH variable stimulates cortisol, the cortisol variable has both a positive and negative feedback on the $\mathrm{CRH}-\mathrm{ACTH}$ variable. Only the circadian rhythm of ACTH and cortisol is seen in the data and explained by the model, thus the ultradian rhythm is not considered. A four dimensional model including the variables CRH, ACTH, cortisol and glucocorticoid receptors (GR) in the pituitary is presented by Gupta et al. [18]. The validation of the model is performed by simulating the cortisol level by feeding experimental human ACTH data into the equation for cortisol. The ACTH prediction of the model is not validated, with the argument that the hypothalamic derived $\mathrm{CRH}$ cannot be measured and simulations of the closed model did not produce oscillations. In recent work by Hosseinichimeh et al. [22], a meta-analysis of five models published before 2015 are performed using the partial prediction method and the authors conclude that the model proposed by Andersen et al. [2] provides the best overall open loop fits. In Mershon et al. [34] the existence of a CRH-oscillator in the isolated hypothalamus was suggested based on experiments and in Gudmand-Hoeyer et al. [17] an elaborated model, specifically including hippocampal dynamics was developed. Following Mershon et al. [34] the ultradian oscillations are driven by a $\mathrm{CRH}$ pulse generator. Non-Linear Mixed Effects (NLME) modeling showed that two parameters influenced ultradian frequency and that amplitude deviated significantly for 29 subjects divided into three groups, hypercortisolemic depressed, hypocortisolemic depressed and a control group. Similarly, a parameter describ- 
ing the elimination of ACTH deviates significantly between the three groups. This parameter influences the mean values of ACTH and cortisol. The model is not included in the meta-analysis by Hosseinichimeh et al. [22] since the publications appeared approximately simultaneously.

The present model is based on an abstract by Ottesen [38] for the IFAC Congress 2011 in Milan where a comprehensive three dimensional model capable of producing realistic circadian as well as ultradian rhythms in the concentrations of $\mathrm{CRH}, \mathrm{ACTH}$ and cortisol was proposed. The model has been analyzed and modified in [42] and further developed and analyzed in [5]. Like the previous model [17], this model was superior to the rest in fitting data. The model is antecedent for the model presented in Section 3 and it generates the circadian rhythm by an endogenous function of time incorporated in the equation for $\mathrm{CRH}$. Unlike the previous considered models, the production of $\mathrm{CRH}$ up-regulates its own production in accordance with experimental evidence reported in the literature [33, 35, 53]. The novelty of the model is the omission of the hippocampal dynamics aiming at a 'minimal model' concept. Except for some second order couplings (inhibitions) the parsimonious principle is followed. The overall idea is to describe the ultradian oscillations by slowly damped (spiral stable) oscillations instead of demanding sustained ultradian oscillations. The model is not included in the metaanalysis by Hosseinichimeh et al. [22], presumably since it was not been published until now.

\section{Novel model of the HPA axis}

\subsection{Data}

The data come from a study described by Carroll et al. [9], where healthy controls and depressive humans were included. Concentrations were measured within ten minute intervals over 24 hours, which highlights the ultradian pulses in both ACTH and cortisol. The main purpose of this model is to describe the circadian and ultradian rhythms seen in data (on time-scales 24 hours and 1-2 hours) and not the small fluctuations which can be considered as noise (on time-scale 10 minutes or less). To facilitate computational fitting of parameters to model output, data were smooted using a moving average over five neighboring points, see Figure 2. The analysis includes normal subjects, i.e. having no known pathologies, disease or abuse, and subjects diagnosed as having hypercortisolemic depression but no other known pathologies, disease or abuse. 


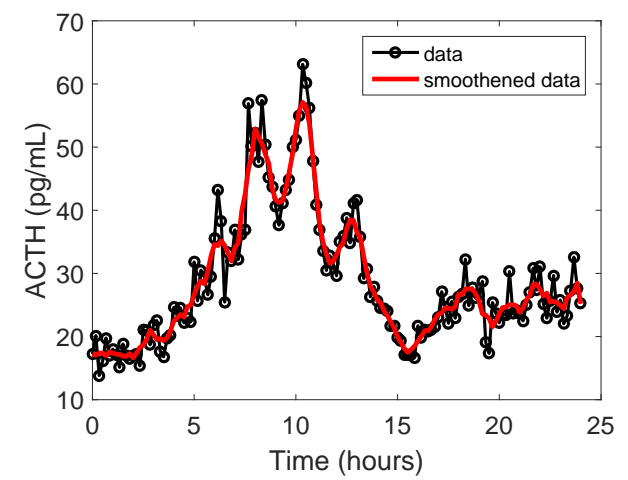

(a)

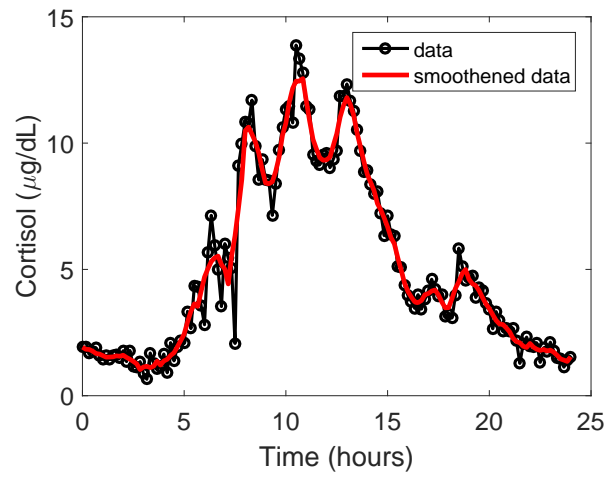

(b)

Figure 2: The raw data are illustrated by the black circles and the red solid line represents the smoothened data, using a moving average filter with a span of five neighboring points.

\subsection{HPA axis model}

A diagram of the model dynamics in Equations (1-4) is illustrated in Figure 1(b) in Section 1. In the model, the rate of change in $\mathrm{CRH}$ is given by two production rates and a first order elimination term (Equation (1)). The production consists of a small baseline production rate and a state dependent rate term. The last is a product of three factors: a circadian function (to be discussed below), an inhibiting cortisol dependent factor (a declining Hill-function) and an auto-up-regulating factor in $\mathrm{CRH}$ itself (Michaelis-Menten expression). [33, 35, 53]. The rate of change in ACTH is described by two terms (Equation (2)): The production rate is inhibited by cortisol, described as a declining Michaelis-Menten expression and is otherwise proportional to $\mathrm{CRH}$, the stimulating ligand. The elimination term is of first order. Finally, the rate of change in cortisol (Equation (3)) is stimulated by the ACTH concentration squared and is exposed to a first order elimination. In contrast to previous models, we allow a positive feedback in hypothalamus, i.e. $\mathrm{CRH}$ is auto-regulated. Another novelty is the inclusion of non-linear terms in the feedback and the feedforward which act synergistically, i.e. large concentrations of ACTH and cortisol affect the production of cortisol and $\mathrm{CRH}$, respectively, more than small concentrations [23]. Finally, a periodically extended bell-like function $C(t)$ models the observed circadian rhythm of the system caused by the 'circadian clock' synchronized by the suprachiasmaticus nucleus $(S C N)$. The ultradian oscillations will manifest itself as weakly damped in response to sudden changes in the circadian rhythm function $(C(t))$, thus no limit cycle is imposed. 
The equations reads,

$$
\begin{aligned}
\frac{d x_{1}}{d t} & =a_{0}+C(t) \frac{a_{1}}{1+a_{2} x_{3}^{2}} \frac{x_{1}}{\mu+x_{1}}-\omega_{1} x_{1} \\
\frac{d x_{2}}{d t} & =\frac{a_{3} x_{1}}{1+a_{4} x_{3}}-\omega_{2} x_{2} \\
\frac{d x_{3}}{d t} & =a_{5} x_{2}^{2}-\omega_{3} x_{3}
\end{aligned}
$$

where $x_{1}, x_{2}$ and $x_{3}$ denote the concentrations of CRH, ACTH and cortisol, respectively, as functions of time. The bell-shaped function

$$
C(t)=N_{c}\left(\frac{t_{m}^{k}}{t_{m}^{k}+\alpha^{k}} \cdot \frac{\left(T-t_{m}\right)^{l}}{\left(T-t_{m}\right)^{l}+\beta^{l}}+\varepsilon\right),
$$

describes the circadian rhythm for $t \in[0, T]$ by the product of two Hill functions. The time unit is minutes and $t_{m}$ can be calculated as $t_{m}=(t-\delta)$ modulo $T$, with $T=1440$ minutes corresponding to 24 hours. Thus, $C(t)$ for $t \in[0, T]$ is extended periodically and shifted by the phase $\delta$. Note $C(t)$ is bounded between $\varepsilon$ and 1 . We will refer to this model as the full model in the following.

\subsection{Parameters}

Parameter values for a hypercortisolemic depressed group of subject and for a control group appear in Table 1 as those for a specific subject appear in Table A.3A.4 in Appendix A. The interpretation of the parameters are as follows,

- $k$ describes the steepness of the increasing Hill-function at the half saturation point $t_{m}=\alpha$, while $l$ descibes the steepness of the decreasing Hillfunction at $t_{m}=\beta$ appearing in $C(t)$. $\varepsilon$ represents the basic contribution from the 'circadian clock', which occurs during the night. $\delta$ describes the time shifting of the circadian rhythm observed in different subjects and $N_{c}$ is a normalization constant.

- The parameter $a_{0}$ is included in the equation for $x_{1}$ to model a basic level of secretion of CRH. This seems reasonable from a biological perspective since it also ensures strict positivity of the model. $a_{0}$ only makes a significant contribution to the system if the concentration of $\mathrm{CRH}$ is low and should thus be set to a small number (it is approximately $7 \%$ smaller than the other rate terms in Equation (1) under normal conditions. The smaller a 
value the more pronounced ultradian oscillations). Whether such small but hypothetical baseline production corresponds to a constant release of CRH or if it represents a background level, e.g. due to lack of well-mixing in the space surrounding the hypothalamus is presently not known.

- $a_{1}$ represents the strength of the auto-up-regulation of $x_{1}$, thus it represents the maximal synthesis of CRH under influence of the 'circadian clock' modeled by $C(t)$ as mentioned.

- $a_{2}$ is multiplied by $x_{3}$ and controls the inhibition of the synthesis of CRH through cortisol. The inhibition of CRH is modeled by a second order term in $x_{3}$. Considering the mechanism as a chemical reaction, this can be interpreted as a cooperative effect. This model choice means that small concentrations of cortisol $\left(x_{3} \ll 1\right)$ has a minor influence, while the effect of large concentrations $\left(x_{3} \gg 1\right)$ is more extensive.

- $\mu$, in the Michaelis-Menten function involving $x_{1}$, is the half-saturation constant. Thus $\mu$ determines the level of $x_{1}$ at which the Michaelis-Menten function will reach half of its saturation value. The Michealis-Menten expression is included to ensure boundness of solutions but makes biological meaning since it reflects that the self-up-regulation mechanism has a saturation.

- $a_{3}$ denotes the strength of the stimulation of $x_{2}$ by $x_{1}$ in the absence of cortisol.

- $a_{4}$ represents the magnitude of the inhibition of the synthesis of ACTH by $x_{3}$.

- $a_{5}$ describes the stimulation of cortisol by ACTH. $x_{2}$ is included in the equation in squared form, which can be interpreted as a cooperative effect in analogy with the inhibition of CRH by cortisol.

- The parameters $\omega_{1}, \omega_{2}$ and $\omega_{3}$ are interpreted as the elimination rates of $\mathrm{CRH}, \mathrm{ACTH}$ and cortisol, respectively. These parameters have been independently examined in several studies and their parameter values are therefore better specified than is the case for the other parameter values. 


\section{Analysis of the model}

\subsection{Trapping region}

The vector field on the right-hand-side of Equations (1-3) is Lipschitz continuous (see proof in Appendix B), hence existence and uniqueness of solution is guaranteed. Thus the existence of attraction trapping regions may be established guaranteeing persistence and boundedness of solutions for any physiologically perturbation.

Let $x=\left(x_{1}, x_{2}, x_{3}\right)^{T}$ be a solution to the system (1-3) and assume that all parameter values are positive and the initial conditions are non-negative. Then positivity of the system and the existence of a positive trapping region, $T_{R}$ follows. Furthermore, this trapping region is attractive, i.e. let $T_{R} \subset\left(\mathbb{R}_{+} \cup\{0\}\right)^{3}$ be the trapping region and $x\left(t_{0}\right) \in\left(\mathbb{R}_{+} \cup\{0\}\right)^{3} \backslash T_{R}$ then $x(t) \in T_{R}$ for $t>t_{1}$, for some $t_{1}>t_{0}$.

To prove the existence of $T_{R}$, first the lower bounds and then the upper bounds are found. The lower bounds also ensure positivity of the system. Consider Equation (1) for $x_{1}=0$, thus $\frac{d x_{1}}{d t}=a_{0}>0$. Hence the solution cannot cross the plane $\left\{x \in \mathbb{R}^{3}: x_{1}=0\right\}$ in negative direction due to the uniqueness of the solution. So for a given $x_{1}\left(t_{0}\right) \geq 0$ then $x_{1}(t)>0$ for all $t>t_{0}$. Then considering Equation (3) for $x_{3}=0$, we have $\frac{d x_{3}}{d t}=a_{5} x_{2}^{2} \geq 0$. Hence $x_{3}\left(t_{0}\right) \geq 0$ implies that $x_{3}(t) \geq 0$ for all $t>t_{0}$ due to the uniqueness of the solution. For $x_{2}=0$ it is observed that $\frac{d x_{2}}{d t}=\frac{a_{3} x_{1}}{1+a_{4} x_{3}}>0$ for all $t>t_{0}$ if $x_{1}>0$ and $x_{3} \geq 0$. Thus it follows from the uniqueness of the solution, that $x_{2}(t) \geq 0$ for $x_{2}\left(t_{0}\right) \geq 0$ and $t>t_{0}$. This in fact implies that $x_{3}(t)>0$ for all $t>t_{0}$. This constitutes the positivity and at the same time constructs the lower bounds of $T_{R}$.

The upper bounds may be found as follows. First consider Equation (1)

$$
\frac{d x_{1}}{d t}=a_{0}+C(t) \frac{a_{1}}{1+a_{2} x_{3}^{2}} \frac{x_{1}}{\mu+x_{1}}-\omega_{1} x_{1} \leq a_{0}+a_{1}-\omega_{1} x_{1} .
$$

where we have used that $C(t)$ is bounded between $\varepsilon$ and 1 . Thus we have that $\frac{d x_{1}}{d t}<0$ for $x_{1}>\frac{a_{0}+a_{1}}{\omega_{1}} \equiv M_{1}$.

Similarly, it follows,

$$
\frac{d x_{2}}{d t}<0 \quad \text { for } \quad x_{2}>\frac{a_{3}}{\omega_{2}} x_{1} \equiv M_{2} x_{1}
$$

and

$$
\frac{d x_{3}}{d t}<0 \quad \text { for } \quad x_{3}>\frac{a_{5}}{\omega_{3}} x_{2}^{2} \equiv M_{3} x_{2}^{2} .
$$


This proves the existence of an attracting trapping region $T_{R}$ where solutions starting outside $T_{R}$ are attracted into the region and solutions inside cannot leave the region. For further details see [5].

Moreover, all the compact sets

$$
T_{R}(\varepsilon)=\left\{x \in\left(\mathbb{R}_{+} \cup\{0\}\right)^{3}: x_{1} \leq M_{1}+\epsilon, x_{2} \leq M_{2} x_{1}+\epsilon, x_{3} \leq M_{3} x_{2}+\epsilon\right\}
$$

for arbitrary $\epsilon \geq 0$ are attracting trapping regions. It is amusing to note, that any solution beginning outside $T_{R}(\epsilon)$ will enter this trapping region in finite time for any $\epsilon>0$. This result follows easily from the fact that continuous functions achieve their minimum on compact intervals. However, the argument may fail in the limit $\epsilon \rightarrow 0$.

\subsection{Dynamics of the system}

The dynamics of the system is investigated for fixed values of the function $C(t)$ describing the circadian rhythm. $C(t)$ is a bounded function, with a slow variation over time compared to the ultradian time-scale of the three variables. This slow time variation suggests an investigation of the system for different fixed values of $C(t)$ in the interval $I=\left[\min _{0 \leq t}\{C(t)\}, \max _{0 \leq t}\{C(t)\}\right]$, for the default parameter values reported in Appendix A. In the following, the system is investigated for fixed values of $C(t)=C_{0} \in I$.

The equilibrium points of the system for constant $C(t)=C_{0}$ are found. Only one real, positive solution, $x^{*}=\left(x_{1}^{*}, x_{2}^{*}, x_{3}^{*}\right)^{T}$, to system (1-3) is found numerically and it depends explicitly on the value of $C_{0}$ [5].

By determining the eigenvalues of the Jacobian matrix at the steady state, it is possible to investigate the stability of the equilibrium. The eigenvalues of the Jacobian matrix for the equilibrium are studied numerically for different fixed values of $C_{0} \in I$ and they are all of the form

$$
\lambda_{1}=-a+i b, \quad \lambda_{2}=-a-i b, \quad \text { and } \quad \lambda_{3}=-c
$$

where $a, b$ and $c>0$. There is one negative eigenvalue and two complex conjugated eigenvalues with negative real part. This means that the equilibria are hyperbolic sink-focuses (spirals) and the points are stable for the nominal parameter values. For further details see [5].

Figure 3 shows the equilibrium for different fixed values of $C(t)=C_{0} \in I$. It is seen that the equilibrium occurs at different positions in the phase space for different choices of $C_{0}$. A solution curve with time-dependent $C(t)$ is also shown in the figure. The figure illustrates how the solution twists around the temporary equilibrium points for fixed $C_{0}$. The solution curve is shown for two days, where the thin gray orbit represents the transient period. 


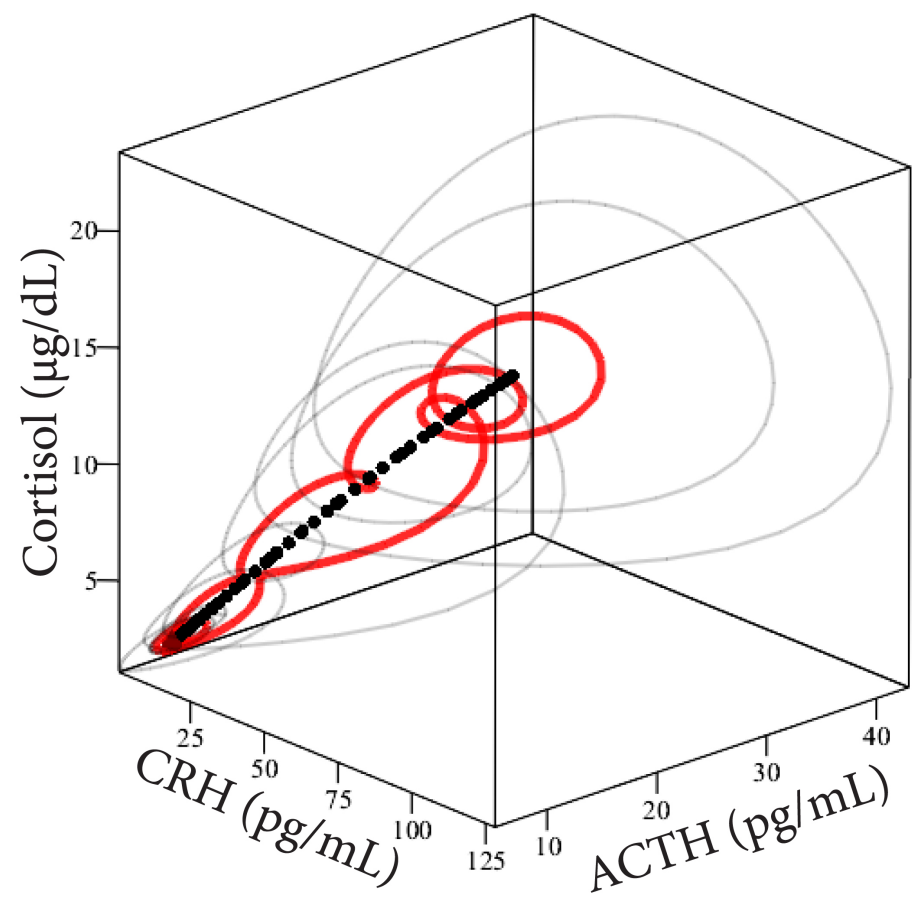

Figure 3: The three dimensional phase space of the HPA system. The equilibrium for fixed values of $C(t)=C_{0} \in I$ are marked by black dots. The orbit represents a solution curve, where the thin gray curve illustrates the transient period and the full red curve the periodic solution.

\section{Results}

\subsection{Sensitivity, subset selection and parameter estimation}

Nominal values for the parameters in Equations (1-3) were found by adjusting these to obtain a model output close to data. Sensitivity analysis was used to analyze if the remaining parameters were sensitive with respect to the model output $\left\{x_{2}, x_{3}\right\}$. Results showed that parameters in $\left\{a_{2}, \mu, a_{3}, a_{4}\right\}$ were insensitive, while parameters in $\left\{a_{0}, a_{1}, a_{5}, \omega_{1}, \omega_{2}, \omega_{3}, \delta\right\}$ were sensitive, see, see Appendix C. Insensitive parameters were kept fixed at their nominal parameter values (given in Appendix A), while sensitive parameters were estimated to minimize the least squares error between model predictions and the data for ACTH and cortisol concentrations as suggested in [37].

In general, it is not possible to show (mathematical) identifiability for non- 
linear ordinary differential equations consequently exact identifiability is not shown. The analysis concludes that it is possible to consider the full model as a model with a reduced number of parameters, denoted the reduced model.

The parameters in the subset are estimated by minimizing the dimensionless, weighted sum of squares

$$
R_{w}=\frac{1}{145} \sum_{i=0}^{144}\left(\left(\frac{A C T H_{i}-x_{2, i}}{\overline{A C T H}}\right)^{2}+\left(\frac{C O R T_{i}-x_{3, i}}{\overline{C O R T}}\right)^{2}\right)
$$

where $A C T H_{i}$ and $C O R T_{i}$ represents the $i$ 'th data point of the relevant subject while $x_{2, i}$ and $x_{3, i}$ are the corresponding model predictions of ACTH and cortisol respectively, at time point $i . \overline{A C T H}$ and $\overline{C O R T}$ denote the mean of the data set for the relevant subject over 24 hours and are used as weights in the function, which is important since the two data sets are of different scales, but fitted simultaneously.

Fits with the optimized parameter for the normal subjects are shown in Figure 4. The simulations of the model for the individual estimated parameters are shown and compared to the raw data and the smoothened data. The hypercortisolemic depressed subjects are treated similar and the graphs for the hypercortisolemic subjects can be found in the Supplementary materials.

In Figure 5, the model output, the standardized residuals against the predicted values, a frequency histogram of the residuals and a QQ plot are shown for the normal subject (f). The histogram and QQ plot suggest that the residuals are indeed normally distributed, while the standardized residuals do not reveal any structure or outliers to cause any major concern. Similar analysis for the other subjects were made confirming the fits (see Supplementary materials).

\subsection{Healthy versus hypercortisolemic depressed groups}

As described in Section 5.1, parameters of the subset selection has been estimated for normal (Control) and hypercortisolemic depressed (Hyper) subjects. Thus the parameter values for the two groups may be compared. More specifically, the hypothesis $\left(H_{0}\right)$ of equal means for the two groups are tested by performing a two-sample $t$-test. The results are shown in Table 1, where the mean value for each parameter for the two groups including their standard deviation, the $p$-values and the outcome of the hypothesis test using a $5 \%$ significance level are reported.

The $t$-tests reveal that three parameters vary between the two groups, namely $a_{0}, a_{1}$ and $\delta$ (actually the $\delta$ parameter was tested by using the circular Gaussian distribution, i.e. von Mises distribution, since the phase is modulo 24 hour). 
Table 1: The values of the estimated parameters and the remaining fixed parameters in the Equations (1-4) for control and hypercortisolemic depressed subjects, respectively. The estimated parameters are shown as the mean and the standard deviation of the estimations. The fixed parameters appear without standard deviation. A hypothesis test $\left(H_{0}\right)$ for equal means are carried out and the $p$-values and the result are shown. A hypothesis value $\left(H_{0}\right)$ of 1 means that the hypothesis can be rejected at a $5 \%$ significance level.

\begin{tabular}{|c|c|c|c|c|c|c|c|}
\hline & \multicolumn{2}{|c|}{ Control } & \multicolumn{2}{|c|}{ Hyper } & $p$-value & $H_{0}$ & Unit \\
\hline$a_{0}$ & $4.71 \cdot 10^{-2}$ & $\pm 4.89 \cdot 10^{-2}$ & $1.31 \cdot 10^{-1}$ & $\pm 8.25 \cdot 10^{-2}$ & $4.47 \cdot 10^{-2}$ & 1 & $\mathrm{pg} /(\mathrm{mL} \cdot \min )$ \\
\hline$a_{1}$ & $6.84 \cdot 10^{12}$ & $\pm 6.89 \cdot 10^{9}$ & $1.29 \cdot 10^{13}$ & $\pm 1.55 \cdot 10^{12}$ & $4.81 \cdot 10^{-5}$ & 1 & $\mathrm{pg} /(\mathrm{mL} \cdot \min )$ \\
\hline$a_{2}$ & $1.78 \cdot 10^{9}$ & & $1.78 \cdot 10^{9}$ & & - & - & $(\mathrm{dL} / \mu \mathrm{g})^{2}$ \\
\hline$\mu$ & 583 & & 583 & & - & - & $\mathrm{pg} / \mathrm{mL}$ \\
\hline$a_{3}$ & $2.28 \cdot 10^{4}$ & & $2.28 \cdot 10^{4}$ & & - & - & $\min ^{-1}$ \\
\hline$a_{4}$ & $1.77 \cdot 10^{5}$ & & $1.77 \cdot 10^{5}$ & & - & - & $\mathrm{dL} / \mu \mathrm{g}$ \\
\hline$a_{5}$ & $3.81 \cdot 10^{-4}$ & $\pm 2.39 \cdot 10^{-4}$ & $3.03 \cdot 10^{-4}$ & $\pm 1.47 \cdot 10^{-4}$ & $4.82 \cdot 10^{-1}$ & 0 & $\frac{\mu \mathrm{g} / \mathrm{dL}}{\min (\mathrm{pg} / \mathrm{mL})^{2}}$ \\
\hline$\omega_{1}$ & $4.49 \cdot 10^{-2}$ & $\pm 1.22 \cdot 10^{-2}$ & $4.57 \cdot 10^{-2}$ & $\pm 1.25 \cdot 10^{-2}$ & $9.04 \cdot 10^{-1}$ & 0 & $\min ^{-1}$ \\
\hline$\omega_{2}$ & $2.25 \cdot 10^{-2}$ & $\pm 1.47 \cdot 10^{-2}$ & $1.46 \cdot 10^{-2}$ & $\pm 4.82 \cdot 10^{-3}$ & $2.16 \cdot 10^{-1}$ & 0 & $\min ^{-1}$ \\
\hline$\omega_{3}$ & $2.01 \cdot 10^{-2}$ & $\pm 1.11 \cdot 10^{-2}$ & $2.10 \cdot 10^{-2}$ & $\pm 5.89 \cdot 10^{-3}$ & $8.67 \cdot 10^{-1}$ & 0 & $\min ^{-1}$ \\
\hline$\delta$ & $8.61 \cdot 10^{2}$ & $\pm 6.91 \cdot 10^{2}$ & $2.01 \cdot 10^{1}$ & $\pm 1.79 \cdot 10^{1}$ & $1.81 \cdot 10^{-2}$ & 1 & $\min$ \\
\hline$\alpha$ & 300 & & 300 & & - & - & $\min$ \\
\hline$k$ & 5 & & 5 & & - & - & - \\
\hline$\beta$ & 950 & & 950 & & - & - & $\min$ \\
\hline$l$ & 6 & & 6 & & - & - & - \\
\hline$\varepsilon$ & 0.01 & & 0.01 & & - & - & - \\
\hline$N_{c}$ & 0.5217 & & 0.5217 & & - & - & - \\
\hline
\end{tabular}

Thus the diagnosed subjects have a disturbed basic production of $\mathrm{CRH}$, auto-upregulation of $\mathrm{CRH}$ and/or circadian phase. 

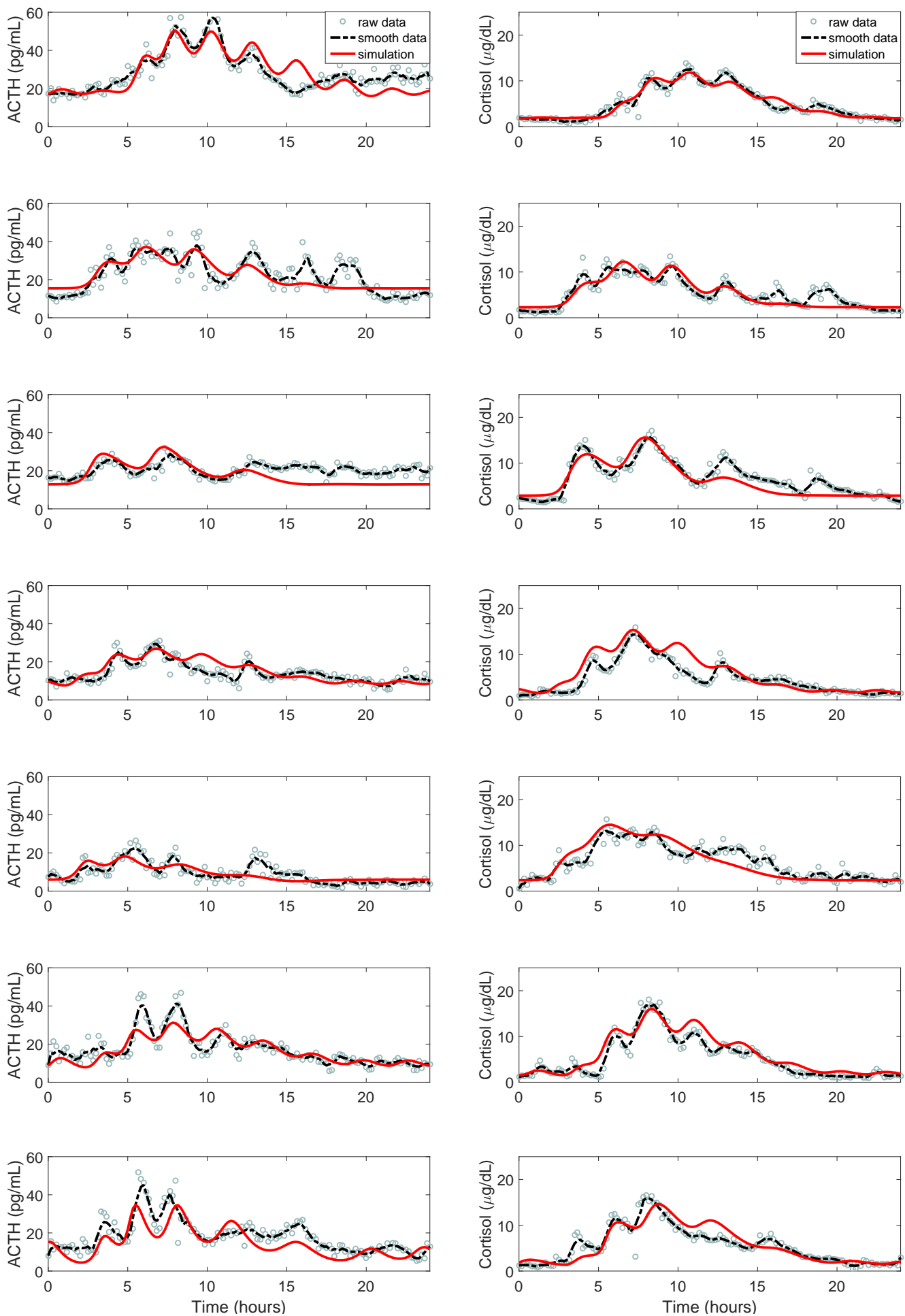

Figure 4: Simulation of the HPA axis model using the estimated parameters. Each row represents a subject, the parameters are estimated individually for each subject.The simulations (red solid lines) are compared to raw and smoothened data for ACTH and cortisol shown in left respective right column (gray circles and black dashed lines 5 respectively). 

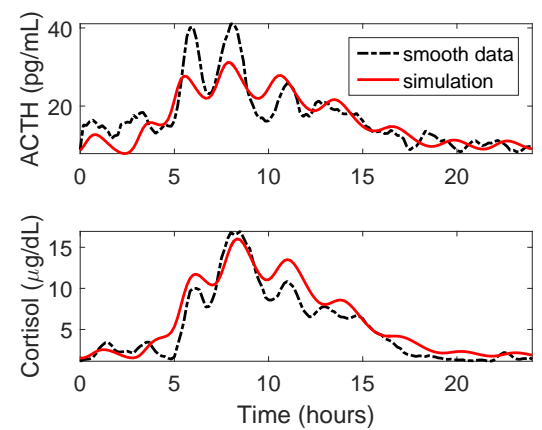

(a)
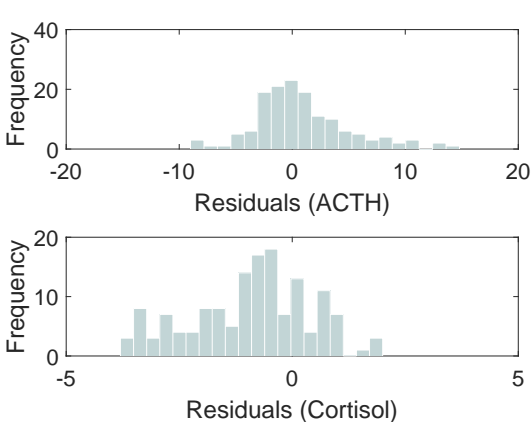

(c)

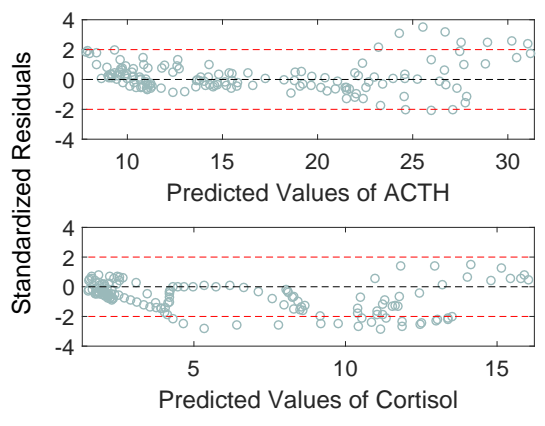

(b)

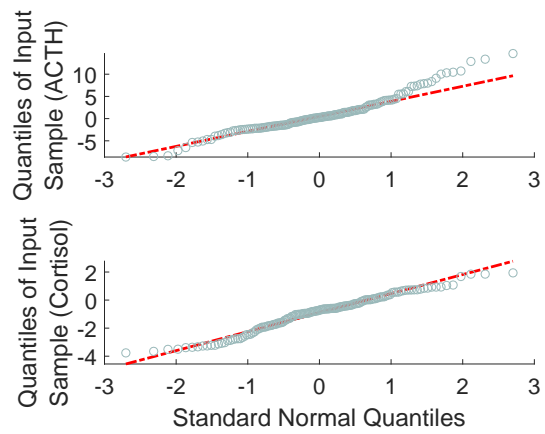

(d)

Figure 5: Residual plots for normal subject (f). The frequency histogram of the residuals and the QQ plot suggest normally distributed residuals and the standardized residuals plotted against the predicted values are structureless and fall in the interval $[-4,4]$. 


\subsection{Approximated model}

In Section 5.1 we noted that insensitive parameters $\left\{a_{2}, \mu, a_{3}, a_{4}\right\}$ and parameters $\left\{N_{c}, k, l, \alpha, \beta, \varepsilon\right\}$ used to predict the bell shaped circadian function were not estimated. Instead of keeping parameters fixed at nominal values, we investigate if the model can be simplified without significantly altering the model output. This means that rather than fixing some parameters in the full model (see Section 5.1) we have performed a model reduction to obtain a fully identifiable model. This model will be referred to as the approximated model.

First we note that $1 \ll a_{2} x_{3}^{2}$ and $\mu \gg x_{1}$ in Equation (1) and $1 \ll a_{4} x_{3}$ in Equation (2) for all times for the nominal values of the parameters. Furthermore, we emphasize that $a_{3}$ is not identifiable since CRH is not measured. Thus $a_{3}$ may vanish by rescaling the $\mathrm{CRH}$ variable leaving the model output for ACTH and cortisol unaltered. Thus system of Equations (1-3) may be approximated by the following simplified equations,

$$
\begin{aligned}
\frac{d y_{1}}{d t} & =b_{0}+b_{1} C(t) \frac{y_{1}}{y_{3}^{2}}-\omega_{1} y_{1} \\
\frac{d y_{2}}{d t} & =\frac{y_{1}}{y_{3}}-\omega_{2} y_{2} \\
\frac{d y_{3}}{d t} & =b_{2} y_{2}^{2}-\omega_{3} y_{3},
\end{aligned}
$$

where $b_{0}=a_{0} a_{3} / a_{4}, b_{1}=a_{1} /\left(a_{2} \mu\right), b_{3}=a_{3} / a_{4}$ and $y_{1}=b_{3} x_{1}$ are the scaled parameters and variable. The unaltered parameters and variables are those appearing in $C(t)$ of Equation (4), the $\omega_{i}$ for $i=1,2,3, b_{2}=a_{5}, y_{2}=x_{2}$ and $y_{3}=x_{3}$. The interpretation of the parameters easily follows from Section 3. A sensitivity analysis of the parameters in the approximated model is provided in Supplementary materials along with a comparison of the reduced and the approximated model in conjunction to data. The analysis shows that all of the parameters of the approximated model are sensitive with respect to the output of the model.

Comprehensive numerical investigations show that the approximated model approximates that of the reduced model. Qualitatively the reduced model calibrates data better than the approximated model: Looking at the figures, the reduced model is most accurate when considering the ultradian oscillations, but the approximated model can be suitable when that is not the main purpose, e.g. when considering the circadian rhythm. Figures showing comparisons of the reduced and the approximated model can be found in Supplemental materials. Quantitatively the models may be compared using their $R_{w}$-values (defined in Equation (6), 
Section 5.1), see Table 2. The $R_{w}$ values suggest that the approximated model is almost as good as the reduced model. As indicated in the third column the variation is relatively large. However, as pointed out above, the reduced model is most accurate when considering the ultradian oscillations.

Table 2: Comparison of $R_{w}$-values of the reduced and the approximated model for the subjects in Supplentary material (in the same ordeR) and the mean over all subjects.

\begin{tabular}{|c|c|c|}
\hline Reduced model $\left(R_{w}\right)$ & Approximated model $\left(R_{w}\right)$ & Percentage of difference \\
\hline 0.139 & 0.123 & -11.4 \\
0.112 & 0.116 & 3.3 \\
0.106 & 0.104 & -2.8 \\
1.051 & 1.580 & 50.3 \\
0.205 & 0.169 & -17.3 \\
0.140 & 0.116 & -17.1 \\
0.307 & 0.243 & -20.8 \\
\hline \hline Reduced model (mean $\left.R_{w}\right)$ & Approximated model (mean $\left.R_{w}\right)$ & Percentage of difference \\
\hline 0.294 & 0.350 & 19.0 \\
\hline
\end{tabular}

\section{Discussion and conclusion}

A novel model of the HPA axis is suggested along with a reduced and an approximated model. The accuracy of the full model is achieved by fitting subjects of a control group and a hypercortisolemic group. This leads to the conclusion, that the model is an adequate model describing the interactions in the human HPA axis. The model has the desirable properties of existence, uniqueness and positivity of the solutions as well as the presence of a positive attracting trapping region. Positivity and existence of an attracting trapping region is a necessary demand for having a physiologically suitable model of the HPA axis. Such findings are confirmed by the simulations as illustrated in Figures 3 and 4, and takes over to the reduced model.

A sensitivity analysis for the full model has been performed, showing that two parameters $\left(a_{5}\right.$ and $\left.\omega_{3}\right)$ are leading and that $a_{3}$ is not identifiable, since $\mathrm{CRH}$ is not observed. The model outcome is also insensitive to variation in the parameters $a_{2}$, $a_{4}$ and $\mu$, hence none of these are practically identifiable [37]. Three parameters are important when trying to fit all data sets, $a_{5}, \omega_{3}$ and $\delta: a_{5}$ changes the ACTH level but does not change the level of cortisol to a greater extend. $\omega_{3}$ has a great impact on the frequency and amplitude of the ultradian oscillations in the outcome for all three variables. Finally, $\delta$ is a highly essential parameter characterizing the 
instance of the circadian peak. The parameters, $\alpha, \beta, k, l$ and $\varepsilon$ (and consequently the derived parameter $N_{c}$ ) entering into the bell-shaped circadian function (Equation (4)) are not subject for parameter estimation in the present investigation. These fixed parameters should ideally be included in the examination of the model but thus the number of parameters exceed what is manageable. Illustrations of the effect to the outcome by varying the parameters and the sensitivity analysis appear in the Supplementary materials.

Using parameter estimation of the seven parameters $a_{0}, a_{1}, a_{5}, \omega_{1}, \omega_{2}, \omega_{3}$ and $\delta$ in the reduced model for healthy and hypercortisolemic depressed subjects gave reliable fits (see Section 5 and Supplementary materials). The resulting model simulations are adequate, with the greatest problem appearing to be the description of the oscillations of the right end tails of both ACTH and cortisol as shown in Figure 4. This is a consequence of the periodicity of the model solutions, which is not fully achieved by the data. Similar most ultradian oscillations in the model become less pronounced than observed in data. This may to some degree be improved by including the buffering effect from the transport molecule CBG. Data and models show that ultradian oscillations are largest at circadian peaks otherwise they tend to vanishes. This is explained by the model as the ultradian oscillations are initiated by a steep increase in the circadian rhythm and as the circadian curve $C(t)$ declines more slowly the ultradian oscillations are damped.

The advantage of the present approach is (1) that the model is simple, thus the parameter estimation runs fairly fast on a computer (approximately 10 minutes per subject), (2) that it is based on empirical facts and in addition is semi-mechanistic in nature, thus each parameter is related to processes for specific glands or specific eliminations, which is an advantage in diagnostics, since it points toward potential pathologies, (3) it is superior in fitting data which is a necessary demand for reliability of the predictions. To our knowledge only one other model share most of the same properties, namely the one presented by Gudmand-Hoeyer et al. [17]. However, that model is based on the physiological paradigm that ultradian oscillation is due to a corticotropin-releasing hormone pulse generator in macaque hypothalamus (referring to Mershon et al. [34]) whereas the model in the present paper does not have an ultradian pulse generator solely located in hypothalamus, a paradigm going back to Engler et al. [14]. Amongst earlier models, only Gudmand-Hoeyer et al. [17] have been able to fit individual data from a group of subjects equally well. Finally, it is considered an advantage that the present method for comparing subpopulations and individuals is fast and fairly straightforward. Using a local optimization algorithm in the parameter estimation procedure runs faster than global methods but may fail to capture the (global) optimum, especially if subjects have 
parameters far away from the nominal values. It is not crucial to have measurements every 10 minutes over 24 hours for using the present methods. However, if one wants to capture ultradian oscillation with a period of 1-2 hours one should at least sample data each 30 minutes.

We notice that our estimates for the elimination rates are 0.0337, 0.0205, $0.0238 \mathrm{~min}^{-1}$ for CRH, ACTH, and cortisol, respectively. For ACTH and cortisol these deviates approximately by a factor two compared with previous estimates using half-life rates. Andersen et al. [2] and Vinther et al. [50] report these to be 0.035 and $0.009 \mathrm{~min}^{-1}$ respectively. Half-life rates are normally measured for ACTH and cortisol by injecting an artificial large dose of the substance. Thus the dynamics are expected to be dominated by the eliminations, which usually are confirmed by semi-logarithmic plot of data. Mathematically this corresponds to neglecting all but the elimination terms in the differential equations resulting in an exponential decline in concentration. Hindmarsh and Charmandari [21] report great variation in half-life rates for cortisol between 48 subjects (up to a factor 3.5). Moreover, for normal values of concentrations of the substances the elimination may be dominated by other mechanisms or non-linearity, e.g. threshold or saturation phenomena may occur. Since our values are indeed confirmed by the declining parts of the ultradian oscillations we rely on our values for the specific subjects considered. For CRH the deviation is approximately a factor 5, but reliable direct measurements of $\mathrm{CRH}$ in the hypothalamic tract in healthy humans is problematic for ethical reasons. Andersen et al. [2] and Vinther et al. [50] give the rough half-life estimate $0.173 \mathrm{~min}^{-1}$ for $\mathrm{CRH}$ based on $\mathrm{CRH}$ measurements reported in [15]. Thus a deviation of a factor 5 is considered acceptable compared to variation for cortisol, which is a factor 3.5. We note that the model in all cases are calibrated to ACTH and cortisol data and emphasize that this gives suitable graphs for CRH concentrations too, see Figure 1 in Section 1.1 of Supplementary material. These are obtained automatically without having addressed any requirement for $\mathrm{CRH}$ predictions. We note, the average value for our $\mathrm{CRH}$ predictions are $15 \pm 8 \mathrm{pg} / \mathrm{ml}$, which is consistent with the rough estimate $7.7 \mathrm{pg} / \mathrm{ml}$ appearing in [15].

By statistical $t$-test on the achieved parameter estimates for hypercortisolemic depressed and controls, three parameters are identified as deviating between the two groups. These are $a_{0}, a_{1}$ and $\delta$. Where the two first are related to the synthesis of CRH the last one relates to the circadian rhythm. We notice that the model differentiates between the groups in agreement with the findings in [39] where mixture modeling and cluster analysis has been used. Hence, it is suggested that the subjective psychological diagnose for hypercortisolemic depression could be 
substituted by testing whether a subject has significant different parameter values for these three parameters. Hereby an objective method for diagnosing is suggested. The method offers a refinement in the diagnosis as well since all combinations of the parameters may appear and suggest individual treatment protocols. In addition, such an objective method based on parameter estimation points out the cause of depression which may be valuable for treatment as well as for the pharmaceutical industry. When applying the model to differentiate between groups it would be preferable to have a large number of subjects in each groups. Thereby the statistical test would improve in quality. However, clinical examination is very expensive and investigations on large cohorts do not exist.

An approximated model is presented which is practical identifiable, see sensitivity analysis in Supplementary materials. Hereby the number of parameters is reduced to seven parameters (i.e. $b_{0}, b_{1}, b_{2}, \omega_{1}, \omega_{2}, \omega_{3}$ and $\delta$ ) and the model becomes practical identifiable given data for ACTH and cortisol. The approximated model still fulfills the positivity condition but the arguments for uniqueness and existence are violated. As a consequence the argument for proving especially upper bounds for the trapping regions for the approximated model is corrupted. An additional mathematical problem is that the equations have singularities as $y_{3} \rightarrow 0^{+}$. However, this may be less critical from a biological point of view. We note that the approximated model lack direct physiological foundation: The amount of cortisol $y_{3}$ appears in the denominators in Equations (7) and (8) and both the elimination and the $\mathrm{CRH}$ auto-up-regulating terms are linear in $y_{1}$. The first means that the production terms tend to infinity as cortisol concentration goes to zero. The second observation means that if $b_{1} / y_{3}^{2}>\omega_{1}$ thus $y_{1}$ will increase toward infinity. This implies that $y_{3}$ have to be greater than $\left(b_{1} / \omega_{1}\right)^{1 / 2}$ for $y_{1}$ to decrease whereas it increase for $y_{3}<\left(b_{1} / \omega_{1}\right)^{1 / 2}$. Both of these behaviors are counteracted by the fact that cortisol production subsequently will increase in response. Comprehensive numerical investigations show that the approximated model has outputs close to the reduced model. Qualitatively the reduced model captures the ultradian oscillations seen in data more accurate than the approximated model. A quantitative comparison of their $R_{w}$-values can be found in Table 2 . The $R_{w}$-values suggest that the models are approximately equally good. If capturing the ultradian oscillations accurately is less interesting the approximated model may be suitable, e.g when interested in the circadian rhythm. 


\section{Acknowledgements}

We would like to thank MD Carroll and MD Veldhuis for granting permission to use their clinical data. Data was de-identified raw and digitized data from a previous study [9]. 


\section{Appendix A. Nominal parameter values for the HPA model}

The nominal parameter values shown in Table A.3-A.4 are taken as those for control subject (f) used for the simulations of the HPA axis model (1-3) presented in Section 4.2.

Table A.3: Parameter values for the HPA axis model in (1-3), presented in Section 4.2 (representing control subject (f)).

\begin{tabular}{|c|c|c|c|}
\hline No. & Parameter & Value & Unit \\
\hline 1 & $a_{0}$ & $3.9031 \cdot 10^{-4}$ & $\mathrm{pg} /(\mathrm{mL} \min )$ \\
2 & $a_{1}$ & $6.8390 \cdot 10^{12}$ & $\mathrm{pg} /(\mathrm{mL} \min )$ \\
3 & $a_{2}$ & $1.7809 \cdot 10^{9}$ & $(\mathrm{dL} / \mu \mathrm{g})^{2}$ \\
4 & $\mu$ & $5.8300 \cdot 10^{2}$ & $\mathrm{pg} / \mathrm{mL}$ \\
5 & $a_{3}$ & $2.2803 \cdot 10^{4}$ & $\mathrm{~min}-1$ \\
6 & $a_{4}$ & $1.7745 \cdot 10^{5}$ & $\mathrm{dL} / \mu \mathrm{g}$ \\
7 & $a_{5}$ & $4.6170 \cdot 10^{-4}$ & $\frac{\mu \mathrm{g} / \mathrm{dL}}{\min (\mathrm{pg} / \mathrm{mL})^{2}}$ \\
8 & $\omega_{1}$ & 0.0337 & $\mathrm{~min}^{-1}$ \\
9 & $\omega_{2}$ & 0.0205 & $\mathrm{~min}^{-1}$ \\
10 & $\omega_{3}$ & 0.0238 & $\mathrm{~min}^{-1}$ \\
\hline
\end{tabular}

Table A.4: Parameter values for Equation (4), used for function creating the circadian rhythm for control subject (f) in the HPA axis model presented in Section 4.2.

\begin{tabular}{|c|c|c|c|}
\hline No. & Parameter & Value & Unit \\
\hline 1 & $\delta$ & 83.8 & $\min$ \\
2 & $\alpha$ & 300 & $\min$ \\
3 & $k$ & 5 & - \\
4 & $\beta$ & 950 & $\min$ \\
5 & $l$ & 6 & - \\
6 & $\varepsilon$ & 0.01 & - \\
7 & $N_{c}$ & 0.5217 & - \\
\hline
\end{tabular}




\section{Appendix B. Lipschitz continuity}

To prove existence and uniqueness of the solution to the Equations (1-3), the right-hand-side must be Lipschitz continuous[29].

Consider the system in Equations (1-3) on the form

$$
\begin{aligned}
\frac{d x(t)}{d t} & =f(x, t) \\
x\left(t_{0}\right) & =x_{0},
\end{aligned}
$$

where $x(t)=\left(x_{1}(t), x_{2}(t), x_{3}(t)\right)^{T}, x\left(t_{0}\right)=x_{0}$ denotes the initial condition at time $t_{0}$ and $f(x, t)$ is a vector containing the right-hand-side of the Equations (1-3). In the following, it is shown that $f(x, t)$ is Lipschitz continuous in $x$ over any domain

$$
\mathscr{D}=\left\{(x, t) \mid\left\|x-x_{0}\right\| \leq \eta, t_{0} \leq t \leq t_{1}\right\},
$$

with $\eta>0$ and $t_{1}>t_{0}$, i.e. there exists a constant $\mathscr{L} \geq 0$ such that

$$
\left\|f(x, t)-f\left(x^{*}, t\right)\right\| \leq \mathscr{L}\left\|x-x^{*}\right\|
$$

for all $(x, t),\left(x^{*}, t\right) \in \mathscr{D}$.

The Lipschitz continuity is shown using the $L^{1}$-norm, since the equivalence of finite-dimensional norms ensures that if $f(x, t)$ is Lipschitz continuous in one norm, it holds for any other norm (only the Lipschitz constant $\mathscr{L}$ may depend on the chosen norm)[29]. Considering the $L^{1}$-norm, it is seen that

$$
\left\|x-x^{*}\right\|_{1}=\left|C R H(t)-C_{R} H^{*}(t)\right|+\left|A C T H(t)-\operatorname{ACTH}^{*}(t)\right|+\left|\operatorname{Cortisol}(t)-\operatorname{Cortisol}^{*}(t)\right|
$$

and

$$
\left\|f(x, t)-f\left(x^{*}, t\right)\right\|_{1}=\left|f_{1}(x, t)-f_{1}\left(x^{*}, t\right)\right|+\left|f_{2}(x, t)-f_{2}\left(x^{*}, t\right)\right|+\left|f_{3}(x, t)-f_{3}\left(x^{*}, t\right)\right| .
$$

Looking at the terms one by one, it can be shown, that $\left\|f(x, t)-f\left(x^{*}, t\right)\right\|_{1}$ is bounded by $\mathscr{L}=\mathscr{L}_{1}+\mathscr{L}_{2}+\mathscr{L}_{3}=a_{1}+\left(2 a_{2}+a_{3} a_{4}+2 a_{5}\right)\left(\eta+\left\|x_{0}\right\|\right)+a_{3}+$ $\omega_{1}+\omega_{2}+\omega_{3}$. The following calculations show how to find $\mathscr{L}_{1}, \mathscr{L}_{2}$ and $\mathscr{L}_{3}$ 
(assuming that all parameter values are positive) :

$$
\begin{aligned}
& \left|f_{1}(x, t)-f_{1}\left(x^{*}, t\right)\right|=\mid a_{0}+C(t) \frac{a_{1}}{1+a_{2} \text { Cortisol }^{2}} \frac{C R H}{\mu+C R H}-\omega_{1} C R H \\
& -\left(a_{0}+C(t) \frac{a_{1}}{1+a_{2}\left(\text { Cortisol }^{*}\right)^{2}} \frac{C R H^{*}}{\mu+C R H^{*}}-\omega_{1} C R H^{*}\right) \\
& \leq\left|a_{1}\left(\frac{1}{1+a_{2} \text { Cortisol }^{2}} \frac{C R H}{\mu+C R H}-\frac{1}{1+a_{2}\left(\text { Cortisol }^{*}\right)^{2}} \frac{C R H^{*}}{\mu+C R H^{*}}\right)-\omega_{1}\left(C R H-C R H^{*}\right)\right| \\
& \leq\left|a_{1} \frac{1}{1+a_{2} \text { Cortisol }^{2}}\left(\frac{C R H}{\mu+C R H}-\frac{C R H^{*}}{\mu+C R H^{*}}\right)\right| \\
& +\left|\frac{\text { CRH }^{*}}{\mu+\text { CRH }^{*}}\left(\frac{1}{1+a_{2} \text { Cortisol }^{2}}-\frac{1}{\left.1+a_{2} \text { (ortisol }^{*}\right)^{2}}\right)\right| \\
& +\left|\omega_{1}\left(C R H-C R H^{*}\right)\right| \\
& \leq a_{1}\left|C R H-C^{2} H^{*}\right|+\left|\frac{a_{2}\left(\text { Cortisol }^{2}-\left(\text { Cortisol }^{*}\right)^{2}\right)}{\left(1+a_{2}\left(\text { Cortisol }^{*}\right)^{2}\right)\left(1+a_{2} \text { Cortisol }^{2}\right)}\right|+\omega_{1} \mid C R H-\text { CRH }^{*} \mid \\
& \leq\left(a_{1}+2 \cdot\left(\eta+\left\|x_{0}\right\|\right) \cdot a_{2}+\omega_{1}\right)\left\|x-x^{*}\right\| \\
& \equiv \mathscr{L}_{1}\left\|x-x^{*}\right\| \text {. }
\end{aligned}
$$

The last inequality holds since

$$
\begin{aligned}
\left|\frac{a_{2}\left(\text { Cortisol }^{2}-\left(\text { Cortisol }^{*}\right)^{2}\right)}{\left(1+a_{2}\left(\text { Cortisol }^{*}\right)^{2}\right)\left(1+a_{2} \text { Cortisol }^{2}\right)}\right| & \leq \mid a_{2}\left(\text { Cortisol }^{2}-\left(\text { Cortisol }^{*}\right)^{2}\right) \mid \\
& \leq a_{2} \mid \text { Cortisol }^{*}-\text { Cortisol }_{\| \text {Cortisol }^{*}+\text { Cortisol }} \\
& \leq a_{2} \cdot\left\|x-x^{*}\right\| \cdot\left(\|x\|+\left\|x^{*}\right\|\right) \\
& \leq 2 \cdot a_{2}\left(\eta+\left\|x_{0}\right\|\right)\left\|x-x^{*}\right\| .
\end{aligned}
$$

Considering the second term

$$
\begin{aligned}
& \left|f_{2}(x, t)-f_{2}\left(x^{*}, t\right)\right|=\left|\frac{a_{3} C R H}{1+a_{4} \text { Cortsiol }}-\omega_{2} A C T H-\left(\frac{a_{3} C_{R H}^{*}}{1+a_{4} \text { Cortisol }^{*}}-\omega_{2} A C T H^{*}\right)\right| \\
& =\mid a_{3} C R H\left(\frac{1}{1+a_{4} \text { Cortisol }}-\frac{1}{1+a_{4} \text { Cortisol }^{*}}\right)+\frac{a_{3}\left(C R H-\text { CRH }^{*}\right)}{1+a_{4} \text { Cortisol }^{*}}-\omega_{2}\left(\text { ACTH }- \text { ACTH }^{*}\right) \mid \\
& \leq a_{3} a_{4} \mid C R H\left(\text { Cortisol }- \text { Cortisol }^{*}\right)\left|+a_{3}\right| C R H-C^{*} H^{*}\left|+\omega_{2}\right| \text { ACTH }- \text { ACTH } H^{*} \mid \\
& \leq\left(a_{3}\left(a_{4}\left(\eta+\left\|x_{0}\right\|\right)+1\right)+\omega_{2}\right)\left\|x-x^{*}\right\| \\
& \equiv \mathscr{L}_{2}\left\|x-x^{*}\right\|
\end{aligned}
$$

and finally, the third term is bounded by

$$
\begin{aligned}
\left|f_{3}(x, t)-f_{3}\left(x^{*}, t\right)\right| & =\mid a_{5} \text { ACTH }{ }^{2}-\omega_{3} \text { Cortisol }-\left(a_{5}\left(A C T H^{*}\right)^{2}-\omega_{3} \text { Cortisol }^{*}\right) \mid \\
& \leq a_{5}\left|A C T H-A C T H^{*}\right|\left|A C T H+A C T H^{*}\right|+\omega_{3} \mid{\text { Cortisol }- \text { Cortisol }^{*} \mid} \\
& \leq\left(2 a_{5}\left(\eta+\left\|x_{0}\right\|\right)+\omega_{3}\right)\left\|x-x^{*}\right\| \\
& \equiv \mathscr{L}_{3}\left\|x-x^{*}\right\| .
\end{aligned}
$$

Hence, $\left\|f(x, t)-f\left(x^{*}, t\right)\right\|$ is bounded by $\mathscr{L}=\mathscr{L}_{1}+\mathscr{L}_{2}+\mathscr{L}_{3}$ which means that $f(x, t)$ is Lipschitz continuous in $x$ over $\mathscr{D}$.

The Lipschitz continuity of $f(x, t)$ over $\mathscr{D}$ implies that there exists an unique solution to the Equations (1-3) at least up to a time $t_{2}=\min \left(t_{1}, t_{0}+\eta / S\right)$ where $S=\max _{(x, t) \in \mathscr{D}}\|f(x, t)\|$, following from the existence and uniqueness theorem[29]. 


\section{Appendix C. Sensitivity analysis}

The sensitivity of the model output $y_{i}$, where $y_{1}=\mathrm{ACTH}$ and $y_{2}=$ cortisol, to the model parameters $\left(\theta_{1}, \ldots, \theta_{q}\right)$ can be predicted from the so called sensitivity matrices

$$
S_{i}=\frac{d y_{i}}{d \theta}=\left[\begin{array}{ccc}
d y_{i} / d \theta_{1}\left(t_{i 1}\right) & \cdots & d y_{i} / d \theta_{q}\left(t_{i 1}\right) \\
\vdots & \vdots & \vdots \\
d y_{i} / d \theta_{1}\left(t_{i k_{i}}\right) & \cdots & d y_{i} / d \theta_{q}\left(t_{i k_{i}}\right)
\end{array}\right] .
$$

where $t_{i j}$ is the $k_{i}$ instance of the $j^{\prime}$ th measurement corresponding to output $i$ (see Supplementary materials for illustration of the time dependent sensitivities). Frequently the matrices $S_{i}$ are stacked upon each other, which is also denoted the sensitivity matrix. The rank of this matrix is denoted the sensitivity rank and tells how many sensitive parameters there are. For comparing sensitivities a dimensionless measure is needed and most frequently one uses relative sensitivities defined as the matrices with entries

$$
s_{i j k_{i}}=\frac{\theta_{j}}{y_{i}} \frac{d y_{i}}{d \theta_{j}}\left(t_{i k_{i}}\right)
$$

whenever well-defined. The two-norm of each column may serve as an absolute measure for comparing the sensitivity of each parameter (see Supplementary materials). In practice one set a small number such that parameters are assumed insensitive whenever their sensitivities are smaller than this small number, see [37]. In this case, a threshold value of $10 \% \cdot \max _{j}\left(\left\|s_{i j}\right\|\right)$ is used. 
[1] U Albrecht. Timing to perfection: The biology of central and peripheral circadian clock. Neuron, 74(2):246-260, 2012.

[2] M Andersen, F Vinther, and JT Ottesen. Mathematical modelling of the hypothalamic-pituitary-adrenal gland (HPA) axis: Including hippocampal mechanisms. Mathematical Biosciences, 246(1). Online http://dx.doi.org/10.1016/j.mbs.2013.08.010:122-138, 2013.

[3] M Andersen, F Vinther, and JT Ottesen. Global Stability in Dynamical Systems with Multiple Feedback Mechanisms. To appear in Advances in Pure Mathematics . Scientific Research Publishing, 2016.

[4] N Bairagi, S Chatterjee, and J Chattopadhyay. Variability in the secretion of corticotropin-releasing hormone adrenocorticotropic hormone and cortisol and understanding of the hypothalamic-pituitary-adrenal axis - a mathematical study based on clinical evidence. Mathematical Medicine and Biology, 25:37-63, 2008.

[5] EO Bangsgaard. Mathematical Modelling of the Dynamic Role of the HPA Axis in the Immune System. Master thesis, Technical University of Denmark, 2016.

[6] Liu Bingzhenga, Zhao Zhenye, and Chen Liansong. A Mathematical Model of the Regulation System of the Secretion of Glucocorticoids. Journal of Biological Physics, 17(4):221-233, 1990.

[7] CL Bowden. Strategies to Reduce Misdiagnosis of Bipolar Depression. Psychiatr. Serv., 52(1):51-55, 2001.

[8] BJ Carroll. Diagnostic validity and laboratory studies: Rules of the game. In In Robins, LN and Barrett, JE (Eds). The validity of psychiatric diagnosis. New York: Raven Press, 1989: 229-245.

[9] BJ Carroll, F Cassidy, D Naftolowitz, NE Tatham, WH Wilson, A Iranmanesh, PY Liu, and JD Veldhuis. Pathophysiology of hypercortisolism in depression. Acta Psychiatrica Scandinavia, 115:90-103, 2007.

[10] George Chrousos. Ultradian, Circadian, and Stress-Related HypothalamicPituitary-Adrenal Axis Activity - A Dynamic Digital-to-Analog Modulation. Endocrinology, 139:437-440, 1998. 
[11] M Conrad, C Hubold, B Fischer, and A Peters. Modeling the hypothalamuspituitary-adrenal system: homeostasis by interacting positive and negative feedback. J. Biol. Phys., 35:149-162, 2009.

[12] M Deuschle, U Schweiger, B Weber, U Gothardt, A Körner, J Schmider, H Standhardt, C-H Lammers, and I Heuser. Diurnal Activity and Pulsatility of the Hypothalamus-Pituitary-Adrenal System in Male Depressed Patients and Healthy Controls. J. Clin. Endocr. Metab., 82(1):234-238, 1997.

[13] T Dickmeis and NS Foulkes. Glucocorticoids and circadian clock control of cell profileration: At the inteface between three dynamic systems. Molecular and Cellular Edocrinology, 331:11-22, 2011.

[14] D Engler, T Pham, JP Liu, MJ Fullerton, and IJ Clarke. Studies of the regulation of the hypothalamic-pituitary-adrenal axis in sheep with hypothalamicpituitary disconnection. II. Evidence for in vivo ultradian hypersecretion of proopiomelanocortin peptides by the isolated anterior and intermediate pituitary. Endocrinology, 127:1956-1966, 1990.

[15] Philip Felig and Lawrence A. Frohman. Endocrinology \& Metabolism, Fourth Edition. McGRAW-HILL, Inc., 2001.

[16] JE Griffin and SR Ojeda. Textbook of Endocrine Physiology, Fifth Edition. Oxford University Press, Inc, 2004.

[17] J Gudmand-Hoeyer, S Timmermann, and JT Ottesen. Patient-specific modeling of the neuroendocrine HPA-axis and its relation to depression: Ultradian and circadian oscillations. Mathematical Biosciences, 257:23-32, 2014.

[18] Shakti Gupta, Eric Aslakson, Brian M Gurbaxani, and Suzanne D Vernon. Inclusion of the glucocorticoid receptor in a hypothalamic pituitary adrenal axis model reveals bistability. Theoretical Biology and Medical Modelling, 4, 2007.

[19] MW Hankins, S Peirson, and RG Foster. Melanopsin: an exciting photopigment. Trends in Neuroscience, 31(1):27-36, 2008.

[20] DE Henley, JA Leendertz, GM Russell, SA Wood, S Taheri, WW Woltersdorf, and SL Lightman. Development of an automated blood sampling system for use in Humans. Med. Eng. Technol., 33(3):199-208, 2009. 
[21] PC Hindmarsh and E Charmandari. Variation in absorption and half-life of hydrocortisone influence plasma cortisol concentrations. Clin. Endocrinol., 82(4):557-561, 2015.

[22] N Hosseinichimeh, H Rahmandad, and Wittenborn AK. Modeling the hypothalamus-pituitary-adrenal axis: A review and extension. Math Biosci., 2015. Oct;268:52-65. doi: 10.1016/j.mbs.2015.08.004. Epub 2015 Aug 12.

[23] AJW Hsueh and PBC Jones. Gonadotropin releasing hormone: Extrapituitary actions and paracrine control mechanisms. Ann. Rev. Physiol., 45:8394, 1983.

[24] S Jelić, Ž Čupić, and L Kolar-Anić. Mathematical modeling of the hypothalamic-pituitary-adrenal system activity. Mathematical Biosciences, 197:173 -187, 2005.

[25] DM Keenan and JD Veldhuis. Cortisol feedback state governs adrenocorticotropin secretory-burst shape, frequency, and mass in a dual-waveform construct: time of day-dependent regulation. Am J Physiol Regul Integr Comp Physiol, 285:950-961, 2003.

[26] DM Keenan, J Licinio, and JD Veldhuis. A feedback-controlled ensemble model of the stress-responsive hypothalamo-pituitary-adrenal axis. Proc. Natl. Acad. Sci. USA, 98(7):4028-4033, 2001.

[27] Vadim Kyrylov, Liudmila A. Severyanova, and Anatoly Zhiliba. The Ultradian Pulsatility and Nonlinear Effects in the Hypothalamic-PituitaryAdrenal Axis. Proceedings of the 2004 International Conference on Health Sciences Simulation (HSS 2004), San Diego, California, USA, pages 103111, 2004.

[28] Vadim Kyrylov, Liudmila A. Severyanova, and Amandio Vieira. Modeling Robust Oscillatory Behaviour of the Hypothalamic-Pituitary-Adrenal Axis. IEEE Transactions on Biomedical Engineering, 52, 2005.

[29] R. J. LeVeque. Finite Difference Methods for Ordinary and Partial Differential Equations: Steady-State and Time-Dependent Problems. Society for Industrial and Applied Mathematics (SIAM), Philadelphia, 2007. ISBN 978-0-898716-29-0. 
[30] SL Lightman, CC Wiles, HC Atkinson, DE Henley, GM Russell, JA Leendertz, MA McKenna, F Spiga, SA Wood, and BL Conway-Campell. The significance of glucocorticoid pulsatility. European Journal of Pharmacology, 583(1-2):255-262., 2008.

[31] Yi-Wei Liu, Zhi-Hong Hu, Jian-Hua Peng, and Bing-Zheng Liu. A Dynamical Model for the Pulsatile Secretion of the Hypothalamo-Pituary-Adrenal Axis. Mathematical and Computer Modelling, 29(4):103-110, 1999.

[32] SW Lockley and RG Foster. Sleep: A very short introduction. Oxford University Press, UK., 2012.

[33] V Louis, R King, and AJ Carrillo. In Vivo and in Vitro Examination of an Autoregulatory Mechanism for Luteinizing Hormone-Releasing Hormone. Endocrinology, 120:272-279, 1987.

[34] JL Mershon, CS Sehlhost, RW Rebar, and JH Liu. Evidence of a Corticotropin-Releasing Hormone Pulse Generator in Macaque Hypothalamus. Endocrinology, 130:2991-2996, 1992.

[35] M Motta, F Piva, and L (1970) Martini. , In The Hypothalamus (Martini, L et al ed). New York: Academic Press, 1970.

[36] N Nader, GP Chrousos, and T Kino. Interactions of the circadian CLOCK system and the HPA-axis. Trends in Endocrinology and Metabolism, 21, 5: 277-286, 2009.

[37] MS Olufsen and JT Ottesen. A practical approach to parameter estimation applied to model predicting heart rate regulation. J. Math. Biol., 67:39 - 68, 2013.

[38] JT Ottesen. Patient specific modelling in diagnosing depression. A 5 page abstract for IFAC conference in Milano 2011, 2011.

[39] JT Ottesen. Etiology and Diagnosis of Major Depresseion - A Novel Quantitative Approach. Open Journal of Endocrine and Metabolic Diseases, 3(2):120-127, 2013. doi: 10.4236/ojemd.2013.32018PublishedOnlinehttp: //www.scirp.org/journal/ojemd.

[40] CM Pariante and SL Lightman. The HPA axis in major depression: classical theories and new developments. Trends. Neurosci., 31(9):464-468, 2008. 
[41] J Rankin, JJ Walker, R Windle, SL Lightman, and JR Terry. Characterizing dynamic interactions between ultradian glucocorticoid rhythmicity and acute stress using the phase response curve. PLoS one, 7(2):pp.10, 2012. e30978. doi:10.1371/journal.pone.0030978.

[42] A Rasmussen, A Fogh, A Niebuhr, E Böttiger, S Furbo, and S Larsen. Matematisk modellering: En simulering af HPA-aksen. A second year student project at Roskilde University, 2015.

[43] RA Sarabdjitsingh, F Spiga, MS Oitzl, Y Kershaw, OC Meijer, SL Lightman, and ER De Kloet. Recovery from Disrupted Ultradian Glucocorticoid Rhythmicity Reveals a Dissociation Between Hor-monal and Behavioural Stress Responsiveness. J. Neuroendocrinol., 22(8):862-871, 2010.

[44] Danka Savić and Smiljana Jelić. A mathematical model of the hypothalamopituitary-adrenocortical system and its stability analysis. Chaos, Solitons \& Fractals, 26:427-436, 2005.

[45] Danka Savić, Smiljana Jelić, and Nikola Burić. Stability of a general delay differential model of the hypothalamo-pituitary-adrenocortical system. IJBC, 16:3079-3085, 2006.

[46] EM Sternberg. Neural regulation of innate immunity: a coordinated nonspecific host response to pathogens. Nature Reviews Immunology, 6:318-328, 2006.

[47] Gerard J. Tortora and Bryan Derrickson. Principles of Anatomy and Physiology, 11 th edition. John Wiley \& Sons, Inc., 2006.

[48] KJ Tracey. The inflammatory reflex. Nature, 420:853-859, 2002.

[49] Johannes D. Veldhuis, Ali Iranmanesh, German Lizarralde, and Michael L. Johnson. Amplitude modulation of a burstlike mode of cortisol secretion subserves the circadian glucocorticoid rhythm. Am J Physiol Endocrinol Metab, 257:6-14, 1989.

[50] F Vinther, A Andersen, and JT Ottesen. The Minimal Model of the Hypothalamic-Pituitary-Adrenal Axis. Journal of Mathematical Biology, 63 (4):663-690, 2010. 
[51] Kurt Vonnegut. Breakfast of Champions. Delacorte Press, United States, 1973. ISBN 0-385-28089-0.

[52] Jean D. Wilson and Daniel W. Foster. Williams Textbook of Endocrinology, Eighth Edition. W. B. Saunders Company, 1992.

[53] M Zanisi, E Messi, M Motta, and L Martini. Ultrashort Feedback Control of Luteinizing Hormone-Releasing Hormone Secretion in Vitro. Endocrinology, 121:2199-2204, 1987.

[54] EE Zhang and SA Kay. Clocks not winding down: unravelling circadian networks. NATURE Reviews Molecular cell Biology, 11:764-776, 2010. 